\title{
Photodynamische Therapie. Von der Therapieoption über einen Therapiestandard zur Standardtherapie? State of the Art und zukünftige Entwicklung
}

\section{R. Braathen}

\author{
From a Therapeutic Option Over Therapy Standard to Standard of Therapy? \\ State of the Art and Future Development
}

\section{Zusammenfassung}

Die topische Photodynamische Therapie mit dem hoch spezifischen Wirkstoff Methylaminolevulinate (MAL) ist eine sich schnell entwickelnde Behandlungsmethode für präkanzeröse und kanzeröse Hautläsionen. Methylaminolevulinate steht in einer standardisierten $16 \%$ Creme (Metvix) mit einer langen Lagerfähigkeit zur Verfügung. Dosisfindungsstudien ergaben die beste therapeutische Wirkung bei präkanzerösen und kanzerösen Hautläsionen bei 16\% MAL, 3 Stunden okklusivem Auftragen und anschließender Belichtung mit rotem Licht. Prospektive vergleichende Studien zwischen Photodynamischer Therapie mit MAL und Kryotherapie bei solaren Keratosen und basozellulären Karzinomen zeigten vergleichbare Ansprechraten für beide Behandlungen, das kosmetische Resultat bei der Photodynamischen Therapie war jedoch bedeutend besser. Um gute klinische Resultate zu erreichen ist es sehr wichtig, dass die zu behandelnden Läsionen gut vorbereitet sind. Krusten und Schuppen müssen mittels Kürettage entfernt werden und bei nodulären basozellulären Karzinomen sollte zuerst kürettiert werden, bevor die Metvix Creme aufgetragen wird. Bei 80\% der Patienten ist die Behandlung schmerz-/problemlos. Gewisse Patienten können jedoch starke Schmerzen verspüren. Durch die Verabreichung von Paracetamol, die Verwendung eines zusätzlichen Ventilators und das Besprühen der Behandlungsstelle mit Wasser während der Belichtung können diese Schmerzen beträchtlich gelindert werden. Die Photodynamische Therapie mit Methylaminolevulinat ist heute eine gut dokumentierte „evidence-based“ Behandlung und ist als solche eine Standardtherapie geworden. Sie ist eine neue, innovative Behandlung, die in Anbetracht der ausgezeichneten kosmetischen Resultate als Therapie der Wahl bei ausgedehnten aktinischen Keratosen, Morbus Bowen und vielen basozellulären Karzinomen betrachtet werden muss.

\section{Abstract}

Topical photodynamic therapy using the highly specific agent, methylaminolevulinate (MAL), is a rapidly evolving treatment procedure for precancerous and cancerous skin lesions. Methylaminolevulinate is available in a standardized $16 \%$ cream (Metvix) with a long shelf life. Dose-finding studies have demonstrated the best therapeutic effect on precancerous and cancerous skin lesions with $16 \%$ MAL, 3 hours occlusive application and subsequent illumination with red light. Prospective studies comparing MAL-photodynamic therapy and cryotherapy of solar keratosis and basocellular carcinomas demonstrated similar response rates for the two treatments but much better cosmetic results with the photodynamic therapy. Preparation of the lesions to be treated is important, one should use a curette to remove crusts and scales and in the case of nodular basocellular carcinomas one should debulk lesions, before applying the Metvix cream. Pains are in about $80 \%$ of cases no problem, but occasional patients can have severe pain, which can be treated with Paracetamol and an additional ventilator and by spraying water at the site of treatment during the illumination. PDT using methylaminolevulinate is today a well documented evidence based treatment and is as such state of the art. It is a new and innovative treatment, which because of its excellent cosmetic results, is the treatment of choice for wide spread actinic keratoses, Bowen's disease and many basocellular carcinomas. 
Einleitung

Photodynamische Therapie von Hautläsionen kann prinzipiell auf zwei verschiedene Arten erfolgen, entweder durch systemische Verabreichung der photosensibilisierenden Substanz oder durch lokale Applikation. Weil die systemische Applikation gewisse Nachteile mit sich bringt, hat sich heute vor allem die lokale Applikation durchgesetzt. Die Entwicklung der lokalen Photodynamischen Therapie in der Dermatologie ist in den letzten zehn Jahren geschehen. Benutzt wurden vor allem Derivate der 5-Aminolevulinsäure (ALA).

Die ersten Hautläsionsstudien wurden mit 5-Aminolevulinsäure, gemischt/gelöst in verschiedenen Cremes und Lösungen sowie in verschiedenen Konzentrationen durchgeführt. Eine Reihe von Kasuistiken und kleinen unkontrollierten Studien sind publiziert worden, bei gewissen Indikationen mit zum Teil sehr guten Resultaten. Weil es keine einheitliche Standardisierung der Behandlung gab, können die Resultate der verschiedenen Studien aber nicht verglichen werden. Die Probleme sind die Konzentration von ALA in der Basiscreme oder Flüssigkeit, die Zusammensetzung der Basiscreme oder der Flüssigkeit, die Haltbarkeit von ALA in den verschiedenen Cremes/Lösungen, die Zeit der Applikation und Okklusion, die Qualität der Lichtquelle sowie Dauer der Belichtung und nicht zuletzt auch die Präparierung der zu behandelnden Läsionen vor der Behandlung.

Photodynamische Therapie mit Methylaminolevulinat (MAL)

Mit der Einführung von standardisierten Präparaten wie z.B. das einzige zur Zeit in Europa erhältliche Arzneimittel, Metvix ${ }^{\circledR}$ Creme, das Methylaminolevulinat (MAL) 16\% enthält, mit einer langen Haltbarkeitsdauer, wurde der erste Schritt zur Standardisierung der Behandlung gemacht. Die Firma PhotoCure hat mit Metvix $^{\circledR}$ eine Reihe von prospektiven randomisierten Multizenter-Studien gemacht.

Zuerst wurden „Dose-Finding“-Studien mit verschiedenen Konzentrationen von MAL und verschiedenen Applikationszeiten durchgeführt. Die Resultate dieser Studien zeigten, dass 16\% MAL sowie 3 Stunden Okklusion und Belichtung mit rotem Licht die besten Resultate ergaben und dies wurde dann als standardisierter Behandlungsmodus in spätere Studien eingesetzt [1 - 17].

In prospektiven vergleichenden Studien zwischen MAL-PDT und Kryotherapie bei solaren Keratosen sowie in prospektiven Studien von basozellulären Karzinomen konnten sehr gute kosmetische Resultate mit PDT erzielt werden, besser als mit Kryotherapie. Sowohl die Patienten als auch die behandelnden Ärzte waren zufriedener mit MAL-PDT als mit Kryotherapie [5-7,12,13]. Bei MAL-PDT von basozellulären Karzinomen empfehlen sich zwei Behandlungen mit einer Woche Zwischenzeit $[10,14,16]$.

Während früher PDT eine Therapieoption war, die vor allem von neugierigen und interessierten Ärzten als experimentelle Therapie durchgeführt wurde, können wir heute sagen, dass diese Therapie durch kontrollierte Studien nun „evidence based“ ist. Dadurch hat sich die Therapie an vielen Kliniken und auch in Privatpraxen zu einer Standardtherapie vor allem bei aktinischen Kera- tosen und Morbus Bowen aber auch bei basozellulären Karzinomen entwickelt. Bei anderen Hauterkrankungen wie z.B. plane Warzen sind ebenfalls gute Behandlungsresultate erreicht worden.

\section{Präparation der Läsionen vor PDT}

Um gute klinische Resultate zu erreichen ist es sehr wichtig, dass die Läsionen gut präpariert sind. Bei aktinischen Keratosen heisst dies, die eventuellen Krusten und Schuppen vor der Behandlung mittels Kürettage zu entfernen. Dasselbe gilt für Schuppen und Krusten bei Morbus Bowen. Bei basozellulären Karzinomen sollte man, falls die Karzinome dicker als $2 \mathrm{~mm}$ sind, so kürettieren, dass der größte Teil des Karzinoms entfernt wird. Dieser Prozess, „Debulking“ genannt, ist sehr wichtig, um eine erfolgreiche Behandlung zu erzielen. Die Blutung nach Debulking stoppt man mit einfacher Kompression. Danach wird eine dicke Schicht Metvix ${ }^{\circledR}$ aufgetragen und vor der Belichtung während mindestens 3 Stunden unter Okklusion auf der Läsion belassen. Die Belichtung sollte mit rotem Licht geschehen. Bei basozellulären Karzinomen sollte die ganze Behandlung nach einer Woche wiederholt werden [2, 8-10,14-17].

Die Präparation der zu behandelnden Läsion bewirkt ein besseres Eindringen des Medikamentes. Wir benutzen eine Prä-PDTCreme ein bis zwei Wochen vor der geplanten Therapie sowie eine Post-PDT Creme nach der Behandlung mit guten Resultaten.

\section{Schmerzen}

Schmerzen sind in ca. 80\% der Fälle kein Problem. Eine Untersuchung bei uns hat gezeigt, dass der Schmerz unabhängig vom Läsionstyp ist, jedoch vom Behandlungsareal abhängig ist. Kopfhaut und Hände sind schmerzempfindlicher als der Körper. Die meisten Patienten finden PDT weniger schmerzhaft als die Kryotherapie und der Effekt von Medikamenten auf den Schmerz zeigte, dass Paracetamol gleichermassen effizient war wie Tramadol, ein Opiumderivat [18]. Ein Ventilator, zusätzlich zu den in den Lampen eingebauten Ventilatoren, scheint einen guten Effekt auf den Schmerz zu haben. Auch Besprühen mit Wasser, z.B. Thermalwasser, ist wirksam, wahrscheinlich durch die Abkühlung verursacht durch die Verdampfung von Wasser, wo $1 \mathrm{~g}$ Wasser 580 Kalorien braucht, die von der Umgebung geliefert werden müssen, was dann zu einer Kühlung und dadurch Schmerzlinderung führt.

\section{Praktische Durchführung der Photodynamischen Therapie}

Wegen der Wartezeit zwischen Auftragen von Metvix ${ }^{\circledR}$ und Belichtung von mindestens 3 Stunden, empfiehlt es sich, die Patienten am Vormittag zu bestellen für das Auftragen der Creme. Die Läsionspräparierung und das Auftragen der Creme ist Sache des Arztes. Dies ist ein sehr wichtiger Teil der Behandlung. Bei tiefen Außentemperaturen darf sich der Patient nicht der Kälte aussetzen, weil dadurch die Produktion von Protoporphyrin 9 in geringem Maße erfolgt. Also muss der Patient in der kalten Jahreszeit im Hause bleiben. Wir geben dem Patienten Kaffee und 
genügend Lesestoff und einige gehen essen. Nach 3 Stunden, aber auch später, kann man die Belichtung vornehmen. Dies wird durch eine ausgebildete Krankenschwester/Praxisgehilfin gemacht. Wir haben gute Erfahrungen mit der Einführung von so genannten PDT-Tagen gemacht, die PDT wird an bestimmten Wochentagen ausgeführt.

\section{State of the Art}

Basierend auf durchgeführten Studien mit standardisierten Präparaten ist die Situation in Europa klar. Das einzige in Europa heute zugelassene standardisierte Medikament ist Metvix ${ }^{\circledR}$ Creme, die 16\% MAL enthält. Die durch wissenschaftliche Studien belegte Medikamenteneinwirkungszeit von 3 Stunden sollte eingehalten werden und die Belichtung sollte mit rotem Licht geschehen [1 - 18]. Es gibt auf dem Markt mehrere Lampen, die einfach zu bedienen sind und mit LED-Technologie ausgestattet sind. Diese „State of the Art“ ist durch die Studien gut „evidencebased“. PDT kann auch beliebig oft wiederholt werden und man verbaut sich auch keine anderen Therapieoptionen, wenn man PDT als erste Therapie wählt.

\section{Zukünftige Entwicklungen}

Das Einsetzen der PDT-Behandlung bei inflammatorischen Dermatosen ist noch kein abgeschlossenes Kapitel. Hier müssen in der Zukunft gut kontrollierte Studien die Wirkung dokumentieren. Die Spezifität von MAL für prämaligne und maligne Läsionen ist gut dokumentiert und die Behandlungsresultate sind so gut, dass es zweifelhaft ist, ob neuere/andere Photosensibilisatoren Vorteile bringen werden in Bezug auf den klinischen Effekt.

Die Schmerzbekämpfung ist ein Gebiet, auf dem man wahrscheinlich etwas erreichen kann. Vielleicht durch die Einführung von Lampen, die mit einer geringen Intensität am Anfang der Behandlung arbeiten, um dann im Laufe der Belichtung die Intensität zu verstärken. Dadurch könnte man erreichen, dass der anfängliche „Burst“ bei der Behandlung, der zu Schmerzen führt, vielleicht etwas gemildert werden kann und die phototoxischen Reaktionen etwas mehr verteilt auf die Zeit geschehen. Die Behandlung von Altershaut generell ist auch ein Thema, das mehrere aufgegriffen haben. Viele Patienten mit ausgebreiteten aktinischen Keratosen erscheinen nach der Behandlung von größeren Arealen jünger. Eine verjüngende Wirkung der Haut durch PDTBehandlung ist nicht auszuschließen, müsste aber in kontrollierten Studien dokumentiert werden.

Photodynamische Therapie der Haut mit MAL ist eine innovative und wertvolle neue Behandlungsform, die aufgrund der guten Behandlungsresultate und den ausgezeichneten kosmetischen Resultaten als Therapie der Wahl bei aktinischen Keratosen, Mb Bowen und vielen basozellulären Karzinomen gelten sollte.

\section{Literatur}

${ }^{1}$ Peng Q, Soler AM, Warloe T, Nesland JM, Giercksky KE. Selective distribution of porphyrins in skin thick basal cell carcinoma after topical application of methyl 5-aminolevulinate. J Photochem Photobiol B Biol 2001; 62: 3 140-145

2 Basset SN, Bachmann I, Pavel S, Saksela S, Johnsson M, Ros AM, Paredes B, Larkö O. A dose-finding study of photodynamic therapy (PDT) with Metvix in basal cell carcinoma (BCC). J Eur Acad Derm Venereo 2000; 1 : Abs 39

${ }^{3}$ Fritsch C, Homey B, Stahl W, Lehmann P, Ruzicka T, Sies H. Preferential relative porphyrin enrichment in solar keratoses upon topical application of $\delta$-aminolevulinic acid methylester. Photochem Photobiol 1998; 68: $218-221$

${ }^{4}$ Braathen LR, Paredes B, Frohlich K. A dose-finding study of photodynamic therapy (PDT) with Metvix in actinic keratosis (AK). J Eur Acad Derm Venereo 2000; 2 Suppl 1: 38

${ }^{5}$ Basset SN, Ibbotson S, Emtestam L, Tarstedt M, Morton C, Maroti M, Calzavara-Pinton PG, Varma S, Roelandts R, Wolf P, Saksela S et al. Photodynamic therapy using Metvix is as efficacious as cryotherapy in BCC with better cosmetic results. A comparison of photodynamic therapy using Metvix with cryotherapy in BCC. J Eur Acad Derm Venereo 2001; 15 Suppl 2: 22

${ }^{6}$ Foley P, Freeman M, Vinciullo G, Spelman L, Murrell D, Weightman W, Anderson C, Reid C, Watson A, Fergin P. A comparison of photodynamic therapy using Metvix with cryotherapy in actinic keratosis. J Eur Acad Derm Venereo 2001; 15 Suppl 2: 22

${ }^{7}$ Szeimies RM, Karrer S, Radakovic-Fijan S, Tanew A, Calzavara-Pinton PG, Zane C, Sideroff A, Hempel M, Ulrich J, Proebstle T, Meffert H et al. Photodynamic therapy using topical methyl 5-aminolevulinate compared with cryotherapy for actinic keratosis: A prospective, randomised study. J Am Acad Dermatol 2002; 47: 258 - 262

8 Wennberg AM, Horn M, Wulf HC, Warloe T, Rhodes I, Fritsch C, Kaufmann R, de Rie M, Wolf P, Stender IM, Soler A et al. Metvix photodynamic therapy in patients with basal cell carcinoma at risk of complications and poor cosmetic outcome after conventional therapy. J Eur Acad Derm Venereo 2001; 15 Suppl 2: 22

${ }^{9}$ Soler AM, Warloe T, Tausjo J, Giercksky KE. Photodynamic therapy of residual or recurrent basal cell carcinoma after radiotherapy using topical 5-aminolevulinic acid or methylester aminolevulinic acid. Acta Oncol 2000; 39: 606-609

${ }^{10}$ Soler AM, Warloe T, Berner A, Giercksky KE. A follow-up study of recurrence and cosmesis in completely responding superficial and nodular basal cell carcinomas treated with methyl 5-aminolevulinatebased photodynamic therapy alone and with prior curettage. $\mathrm{Br} \mathrm{J}$ Dermatol 2001; 145: 467-471

${ }^{11}$ Gardlo K, Ruzicka T. Metvix PhotoCure. Current Opinion in Investigational Drugs 2002; 3: $1672-1678$

${ }^{12}$ Freeman M, Vinciullo C, Francis D, Spelman L, Nguyen R, Fergin P, Thai KE, Murrell D, Weightman W, Anderson C, Reid C, Watson A, Foley . A comparison of photodynamic therapy using topical methyl aminolevulinate (Metvix) with single cycle cryotherapy in patients with actinic keratosis: a prospective, randomised study. J of Dermatol Treat 2003; 14: 99-106

${ }^{13}$ Pariser DM, Lowe NJ, Stewart DM, Jarratt MT, Lucky AW, Pariser RJ, Yamauchi PS. Photodynamic therapy with topical methyl aminolevulinate for actinic keratosis: Results of a prospective randomised multicenter trial. J Am Acad Dermatol 2003; 48: 227 - 232

${ }^{14}$ Rhodes L, de Rie M, Enström Y, Groves R, Morken T, Goulden V, Wong G, Grob JJ, Varma S, Wolf P. A randomised comparison of excision surgery and PDT using methyl aminolevulinate in nodular BCC. Ann Dermatol Venero 2002; 129: S108

${ }^{15}$ Wennberg AM et al. Metvix Photodynamic therapy in patients with basal cell carcinoma at risk of poor cosmesis. EADV October 2001, Munich, Poster

${ }^{16}$ Basset-Seguin $\mathrm{N}$ et al. Photodynamic therapy using methyl aminolevulinate is as efficacious as cryotherapy in primary superficial basal cell carcinoma but with better cosmetic outcome. 61st Annual AAD meeting, March 2003, San Francisco, Poster

17 Vinciullo C et al. Photodynamic therapy with methylaminolevulinate $160 \mathrm{mg} / \mathrm{g}$ cream in patients with basal cell carcinoma with a risk of complications and poor cosmetic outcome using conventional therapy. 9th World Congress on Cancers of the Skin, May 7-10th, 2003, Sevilla, Poster Abstract

${ }^{18}$ Kapur N, Kernland LangK, Braathen LR. Photodynamic therapy-induced pain: a patient-centered survey. Br J Dermatol; Annual Meeting the British Association of Dermatologists 2003, Abstract 